\title{
THE 1990 NOBEL PRIZE
}

\section{Finding The Quark}

\author{
M. Jacob
}

\author{
CERN, Geneva, Switzerland
}

The 1990 Nobel Prize for Physics has been awarded jointly to Jerome Friedman and Henry Kendall of the Massachusetts Institute of Technology (Cambridge, USA), and to Richard Taylor of the Stanford Linear Accelerator Center (Stanford, USA) for their key contributions to a series of deep inelastic scattering experiments of electrons on protons and bound neutrons that confirmed the existence of an inner structure in these two constituent particles of the nucleus. The results of what is known as the SLAC-MIT experiment were first disclosed at the 1968 international high energy physics conference in Vienna. They were subsequently interpreted as experimental evidence for quarks, a new layer in the structure of matter, that had been postulated a few years before by M. Gell-Mann and G. Zweig but of which no direct trace had been found.

The quark structure of the proton, involving point-like coloured quarks bound by the colour force into a colourless particle, lies at the root of our present knowledge of the deep structure of matter. It is the origin of a number of recent important findings and remains the framework within which much research is conducted today. Many physicists thus consider the Nobel laureates' pioneering experiments as standing alongside the classical scattering experiments of Rutherford in the early 1900 's that led to the nuclear model of the atom.

\section{The SLAC-MIT Experiment}

The SLAC-MIT experiment was a continuation of earlier work that used electrons to probe the structure of nucleons. It exploited the new, two mile long linear electron accelerator, giving

Maurice Jacob is Head of Theory Division, CERN, CH-1211 Geneva 23, Switzerland. He is a member of the Swedish Academy of Sciences and a Correspondent of the French equivalent. After serving as the President of the French Physical Society in 1985, he became Secretary of EPS in 1986. As a theoretical high energy physicist interested mainly in helicity formalism and jets in hadronic collisions, he has been with the research staffs at Brookhaven, Caltech and SLAC. the highest energies then available, that had been completed in 1965 under the leadership of W. Panofsky, who was SLAC's Director at the time. The experiments employed large magnetic spectrometers to measure the scattering angle and energies of the recoil electrons emerging from the violent collision of the 4 to $21 \mathrm{GeV}$ incident beam with a fixed target comprising either liquid hydrogen or deuterium. The prizewinners collaborated with others in building three spectrometers and they operated two of them - one for scattering angles of $6^{\circ}$ and $10^{\circ}$, the other for angles of $18^{\circ}, 26^{\circ}$ and $34^{\circ}$ (Fig. 1).

The SLAC-MIT collaboration had started in 1967, with the California Institute of Technology as the third partner, with a study of of elastic scattering of electrons against protons to measure the form factors of the nucleons. Previous results at lower energies had shown that the nucleon behaved like a "soft" structure in mainly scattering electrons at small angles. After this initial phase, the MIT-SLAC team turned to inelastic electron scattering where the nucleon target is shattered. Such processes had been identified in experiments at lower energies and nothing very new was expected. The experiment was difficult, the size and com- plexity of the equipment notwithstanding, since cross-sections were a priori very small and important radiative corrections had to be dealt with. The names R. Taylor, J. Friedman and $\mathrm{H}$. Kendall clearly stood out among the researchers who found that the probability of deep inelastic scattering, where the electron looses a large fraction of its energy and emerges at a high scattering angle, was much greater than expected. The results were surprising to many as the proton appeared to be behaving as made up of point-like objects carrying the proton's global positive charge, and responding independently to the high energy impinging electrons.

\section{Scaling}

The interpretation in terms of pointlike scatterers followed from the scaling property which was observed. When describing the properties of the scattered electron one has to consider two independent Lorentz invariant variables, namely $m v$ the proton mass times the energy transferred by the electron, and $q^{2}$ the momentum transfer squared. The dynamics, which depends a priori on both, was found to depend only on the ratio $2 m v / q^{2}$ at the large values of $v$ and $q^{2}$ which could be reached simultaneously at SLAC. This property, called

Fig. 1 - The two magnetic spectrometers used for the SLAC-MIT experiment. The $8 \mathrm{GeV}$ spectrometer is in the foreground and the $20 \mathrm{GeV}$ unit is to the rear. The bulk of the detectors comprise shielding (weighing 450 tons for the $8 \mathrm{GeV}$ device).

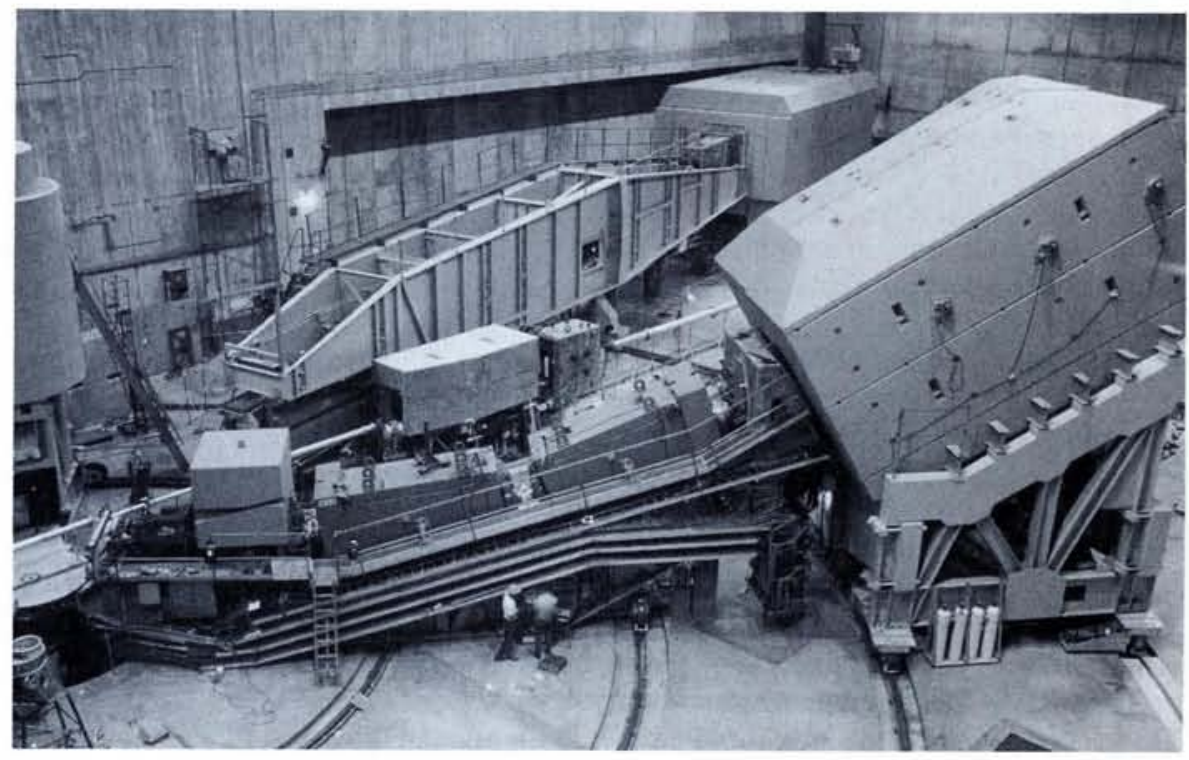




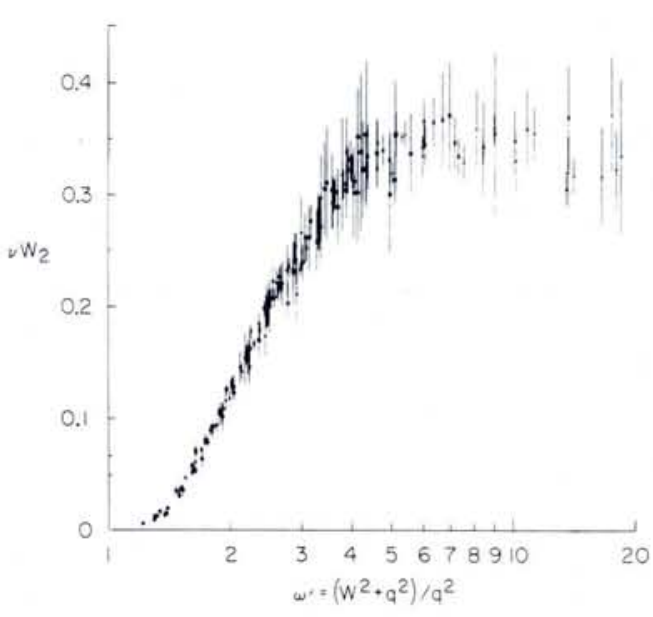

"scaling" (Fig. 2), is what is expected for scattering on point-like constituents within the target proton. The 1990 Nobel Prize has been awarded for this remarkably clear result from a difficult experiment.

J.D. Björken, a theoretician at SLAC, had in fact predicted in 1967 the scaling behaviour long before the results of the SLAC-MIT experiment were available.
Fig. 2 - The scaling behaviour of deep inelastic scattering of electrons on protons. Data from the SLAC-MIT experiment showing that the structure function $W_{2}$ where $v$ is the transferred energy, depends only on the ratio $2 \mathrm{mv} / \mathrm{q}^{2}$ where $m$ is the proton mass and $q$ is the transferred momentum. This behaviour is expected if scattering occurs on point-like constituents in the target nucleons. From Miller G. et al., Phys. Rev. D5 (1972) 528 with permission.

He used a tool called Current Algebra which had been developed as a theoretical device for attaining wide-ranging properties. The quark theory that could be used was considered too elementary to be correct and it invoked quark fields for which no particles were known. Yet there were strong reasons to believe that the Current Algebra relations which could be thus derived were far more general than the theory within which they were obtained.

The quark structure of matter itself dated back to the early sixties and the success of the "eightfold way" of M. Gell-Mann and Y. Ne'eman. Large numbers of particles called hadrons, which resembled nucleons or $\pi$-mesons in some respects, had been discovered. The similarities and differences among these hadrons could be accounted for in terms of Unitary Symmetry, and the simplest visualization of the abstract mathematical description became much more comprehensible when $\mathrm{M}$. Gell-Mann and G. Zweig proposed that hadrons could be "constructed" in terms of building blocks called quarks for the baryons, and a quark-antiquark pair for the mesons The properties of these hypothetical particles, and in particular their fractional charges, were however such that few believers came forward. Quarks, moreover, had been sought but never found.

Whilst Björken's approach was carefully phrased in terms of the properties
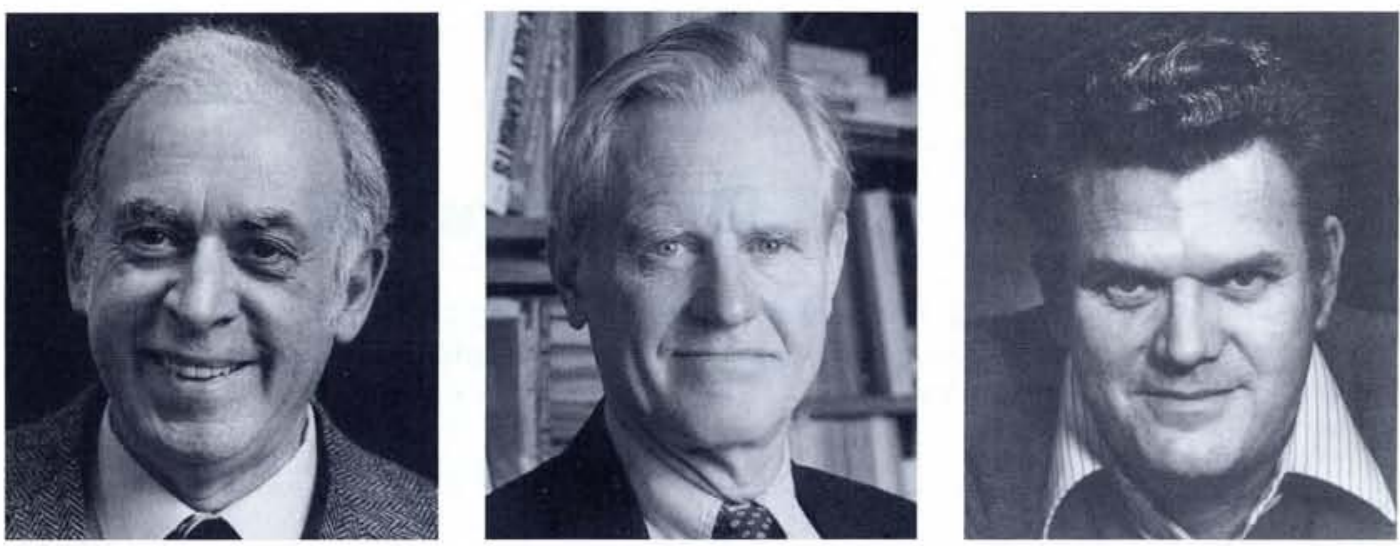

The 1990 Nobel laureates: Jerome Friedman, Henry Kendall and Richard Taylor (from left to right).

Jerome I. Friedman was named last year as the William A. Coolidge Professor of Physics in the Laboratory for Nuclear Science (LNS), Massachusetts Institute of Technology, Cambridge, MA, USA. Professor Friedman was born in 1930 in Chicago and graduated from the University of Chicago where he stayed for one year before becoming a Research Associate at Stanford University in 1957, and an Associate Professor at MIT in 1960. Apart from his contributions to the SLAC-MIT experiment, he is also well known as the co-discoverer of the violation of parity conservation in the decay of the $\pi$-meson. Awarded the W.K.H. Panofsky Prize jointly with the other Laureates in 1989, Professor Friedman is a Fellow of the American Physical Society and a Member of the American Academy of Arts and Sciences. He has been the Director of the LNS (1980-83) and Head of MIT's Physics Department (1983-88), and he is currently serving on the Scientific Policy Committee of the Superconducting Super Collider Laboratory, TX, USA.

\section{The 1990 Nobel Laureates}

Henry W. Kendall has been a Professor of Physics in MIT's Laboratory for Nuclear Science since 1967. Born in Boston in 1926, he graduated from MIT in 1955 and then worked as a National Science Foundation postdoc at the Brookhaven National Laboratory and MIT for three years. Following six years in the Physics Department, Stanford University, he joined the MIT faculty in 1961. He is a founding member of the Union of Concerned Scientists (UCS) and in this capacity has served on various panels dealing with reactor safety. $\mathrm{He}$ has been the Chairman of the UCS Board of Directors since 1973 and a member of the Board of Directors of the Arms Control Association since 1979 . He is also a Fellow of the APS and the AAAS and received the Leo Szilard Award and the Bertrand Russell Society Award in 1982
Richard E. Taylor is Professor of Physics at the Stanford Linear Accelerator Center (SLAC), Menlo Park, CA, USA. Born in Alberta, Canada in 1929, he graduated with an M.Sc. from the University of Alberta in 1952 and with a Ph.D. from Stanford University in 1962. After holding research positions at both the Laboratoire de I'Accélérateur Linéaire, Orsay, France and the Lawrence Radiation Laboratory, Berkeley, CA, USA, he joined the SLAC staff, becoming Professor in 1968 and serving as the Associate Director for Research from $1982-86$. He is involved in the preparation of one the HERA experiments at DESY, Hamburg which will move into the beam next August, and he served on the LEP Experiments Committee at CERN, Geneva until last year. He is a Fellow of the APS and of the Royal Society of Canada. 
of almost equal time commutators at infinite momentum - the accepted orthodox approach - he could not resist relating the inferred scaling property to the possible presence of pointlike constituents, something which was then meeting strong resistance from many. $\mathrm{He}$ has been given much credit by the Nobel laureates for his rôle in helping the experimentalists analyze their data and assess its implications.

The part played by R.P. Feynman at the time is also of great significance. Starting from a different corner of particle physics, namely the properties of particle production in high energy hadron collisions, he had reached the idea that hadrons were made of point-like partons. Thanks to his inspiring talks, these partons were soon recognized by many as the agents at the origin of the scaling property. They were later identified with quarks. To quote Riordan in his book "The Hunting of the Quark", "partons swept through SLAC like a brushfire" after Feynman's first presentation. Feynman could explain scaling in a language which everybody understood. But perhaps more important was the respectability he brought to an idea which seemed unorthodox to many.

\section{Eliminating Doubt}

The results of the SLAC-MIT inelastic scattering experiment were at first believed to be misinterpreted owing to possible errors introduced mainly by the radiative corrections that took account of the energy that an incident or outgoing electron could radiate as light. The scaling property eventually helped a great deal in making the observed rates much higher than those anticipated from a "soft" proton with a charge spread over its volume. By 1968 the statistics were such that it was clear that a major new effect had been found.

It still took a considerable effort by the same team to fully establish the scaling property through an extension of their analysis to larger angles, and therefore lower cross-sections, and to eliminate experimentally all other "explanations" which were of course readily ventured to avoid the need for point-like structures.

The behaviours of the two independent structure functions which contain the dynamical part of the scattering cross-section had to be separated. Scattering off deuterons had to be done in addition to the initial experiments on hydrogen to compare the proton and neutron responses in terms of these structure functions. By 1972, the pointlike constituents were there to stay; they could also be identified with quarks. Moreover, CERN's experiments on deep inelastic neutrino scattering in the Gargamelle bubble chamber started to provide strong supporting evidence for the identification of the point-like scatterers as quarks.

\section{Into The Future}

We understand today how each of the quark types with their different flavours exists in three states differing by a property called colour. The quarks interact strongly via the exchange of massless vector bosons (or gluons) which themselves exist in eight colour states, and the quantum field theory of quarks and gluons, called Quantum Chromodynamics (QCD), represents the modern approach to the strong interaction. Quarks have never been detected as free particles owing to the long-distance nature of the strong force between coloured particles which confines quarks within the colourless hadrons that are observed. We appreciate therefore how coloured objects like quarks can form colourless hadrons and yet never appear as free particles. One also understands how the scaling property emerges at large transferred energy and large $q^{2}$, but not as an exact property since logarithmic deviations originating from the radiation of gluons by the scattered quarks have to be acknowledged.

With the great steps forward of the seventies and early eighties, and the successes of the Standard Model, the electroweak theory of Glashow, Salem and Weinberg combined with QCD, which recently emerged with flying colours from the first round of LEP experiments (see Europhysics News 21 (1990) 166), one now understands rather well the deep structure of hadrons in terms of point-like quarks. Indeed, many predictions thus inferred have been verified. Hadrons containing at least one quark or antiquark of the first five flavours have been observed. The sixth quark, the heaviest called "top", remains unidentified (see page 203) and collisions between quarks in future colliders are expected to probe much deeper into the structure of matter (see below). And all of these past and future developments started with the beautiful experimental result of 1968 which has now been acknowledged with the 1990 Nobel Prize in Physics.

\title{
LHC Physics
}

\author{
CERN's Theory Division looks beyond LEP and the Standard \\ Model in describing the physics potential of a future proton- \\ proton collider.
}

The LEP accelerator at CERN is now fully operational so it is legitimate to prepare for the steps beyond the machine's further development (which is in progress and mainly involves boosting the energy to $200 \mathrm{GeV}$ by installing superconducting RF cavities). The underlying rationale is clear-cut - to reach a more profound understanding of the way the world is built and works, physicists seek greater unity and simplicity in descriptions of the physical world by probing the structure of matter to increasing depth. For behind the enormous variety and complexity of structures met in our everyday experience, there is a basic description in terms of fundamental constituents and interactions. At today's level of scrutiny of $10^{-18} \mathrm{~m}$ we have found quarks and leptons - fundamental point-like components. Their in teractions as summarized by the Standard Model are the building blocks of matter - hadrons, nuclei and eventually atoms and molecules - and they show a cohesion never met before in physics.

The Standard Model is a relatively recent achievement which must be thoroughly tested. CERN'S LEP machine that began operating in July 1989 was designed with this in mind, by continuing with greater accuracy the exploration started at the organization's $\bar{p} p$ collider. For according to the quantum rules governing sub-atomic physics, the price to pay for resolution is energy. So to probe in detail the structure of matter at the level of $10^{-18} \mathrm{~m}$ one needs the 100 to $200 \mathrm{GeV}$ provided by LEP. The physics we meet is the one which prevailed when the expanding Universe was a tenth of a nanosecond old. It is only in this distant past that the many questions raised by the observation of the Cosmos at large can find their answers.

Despite the Standard Model's relative simplicity it is not the ultimate in physics. A natural progression in expanding the model is an order of magnitude increase in resolution, with an analysis of the structure of matter at the level of $10^{-19} \mathrm{~m}$ and, accordingly, an understanding of the physics of the Universe when it was $10^{-12} \mathrm{~s}$ old.

\section{Symmetry Breaking}

In both the electroweak theory, which provides a unifying framework to such diverse phenomena as electromagnetism and radioactivity, and modern descriptions 